\title{
Prospective Evaluation of Doppler Sonography to Detect the Twinkling Artifact Versus Unenhanced Computed Tomography for Identifying Urinary Tract Calculi
}

Ania Z. Kielar, MD, FRCPC, Wael Shabana, MD, PhD, Maryam Vakili, MD, Jonathan Rubin, MD, PhD

Received December 13, 2011, from the Department of Radiology, Ottawa Hospital, Ottawa, Ontario, Canada (A.Z.K., W.S., M.V.); and Department of Radiology, University of Michigan, Ann Arbor, Michigan USA (J.R.). Revision requested December 28, 2011. Revised manuscript accepted for publication April 4, 2012.

We thank Lourdes Garcia and Rebecca Thornhill for help with statistical calculations.

Address correspondence to Ania Z. Kielar, $M D$, FRCPC, Department of Radiology, Ottawa Hospital, C-1, 1053 Carling Ave, Ottawa, ON K1R 4E9, Canada.

E-mail:aniakielar@gmail.com

Abbreviations

BMI, body mass index; CI, confidence interval; $C T$, computed tomography; ED, emergency department; PPV, positive predictive value
Objectives - The twinkling artifact is an emerging tool for identifying urinary tract calculi. The purpose of this prospective study was to evaluate the diagnostic accuracy of the twinkling artifact compared to unenhanced computed tomography in detecting urolithasis.

Methods - After Research Ethics Board approval, 51 patients with flank pain from the emergency department were enrolled between November 2009 and September 2010. Patients received an unenhanced computed tomographic scan with 1.25 -mm raw data and reformatted 5-mm axial and 2-mm coronal images. Blinded assessment of the urinary tract was performed with gray-scale and color Doppler interrogation. The number of calculi, location, size, kidney distance from the skin, body mass index of the patient, and sonographic image parameters were recorded.

Results - There were 35 right-sided and 38 left-sided renal calculi, 14 right-sided and 21 left-sided ureteric calculi, and 6 bladder calculi (total, 114 calculi). Thirteen patients had no calculi. The average calculus size was $2.6 \mathrm{~mm}$ (range, $1-9 \mathrm{~mm}$ ). There were 6 false-positive and 22 false-negative instances of twinkling artifacts. On gray-scale evaluation looking for an echogenic focus with shadowing, there were 8 false-positive and 40 false-negative findings. The positive predictive value (PPV) of the twinkling artifact for identifying calculi was $94 \%$, and the sensitivity was $83 \%$. The PPV of gray-scale sonographic shadowing was only $64.9 \%$, and the sensitivity was $80.2 \%$.

Conclusions - The twinkling artifact has a high PPV for detecting renal and urinary tract calculi. Evaluation for the twinkling artifact is a complementary technique to standard gray-scale shadowing of calculi and improves detection of urolithiasis on sonography.

Key Words — positive predictive value; sonography; twinkling artifact; urinary tract calculi 
The phenomenon was first described by Rahmouni et al ${ }^{1}$ in 1996 as an artifact generated by a strongly reflecting medium composed of small individual reflectors. The twinkling artifact occurs with urinary tract calculi and parenchymal calcifications, but it can also be observed with noncalcified biliary calculi and any material with an irregular, rough, or reflective surface. ${ }^{2}$

In this prospective study, the aim was to test the diagnostic accuracy of the Doppler twinkling artifact for detecting urolithiasis using unenhanced computed tomography (CT) as the reference standard.

\section{Materials and Methods}

We conducted a Health Insurance Portability and Accountability Act-compliant study. After approval of our institute's Research Ethics Board, all of the enrolled patients provided written consent. For inclusion, an unenhanced CT scan of the abdomen and pelvis was requested by an emergency department (ED) physician for flank pain. Patients were enrolled before undergoing the CT scan; therefore, when performing the subsequent sonographic examination, it was not known whether there would be a calculus.

\section{Computed Tomographic Technique}

All CT scans were performed on a 64-slice CT scanner (Lightspeed VCT; GE Healthcare, Milwaukee, WI). All scans were performed with a low-dose, unenhanced "renal colic protocol." The images were sent to a picture archiving and communication system at the original axial 1.25$\mathrm{mm}$ slice thickness in addition to the reconstructed 5-mm axial images and 2-mm coronal images. The $1.25-\mathrm{mm}$ raw data were reviewed to eliminate the possibility of missing small calculi because of volume averaging. The postprocessing techniques do not expose the patient to any additional radiation. One of 2 attending radiologists evaluated the CT images while the sonographic examination was being performed by a sonographer in a blinded fashion. The radiologist filled out a standardized form for the CT findings, indicating the location, size and number of calculi as well as the distance from the skin surface to the renal parenchyma. The sonographer filled out an equivalent form for the sonographic findings to be able to compare similarities and differences in imaging results between the two techniques.

\section{Sonographic Technique}

Right after the CT scan, the patient directly underwent a limited sonographic scan of the kidneys, ureters, and blad- der (iU22; Philips Healthcare, Bothell, WA). This examination was performed for research purposes and was not considered the usual standard of care. It was performed with a standard ultrasound unit, which is always situated in the emergency radiology department, in a dedicated room next to the emergency radiology CT scanner. The examination was performed by a trained sonographer using a curved low-frequency probe $(2-5 \mathrm{MHz})$ and a high pulse repetition frequency, with the machine's scale in the range of 60 to $70 \mathrm{~cm} / \mathrm{s}$. The pulse repetition frequency is defined as the number of pulses sent per second. The use of a high pulse repetition frequency is a key technical factor for identifying and documenting the twinkling artifact. The sonographer was blinded to the results of the CT scan. The sonographers involved in this study have between 3 and 15 years of experience. This process was selected to reflect the nature of daily clinical practice at our institution where sonographers typically perform the initial sonographic examinations. All of the sonographers in our hospital were trained with an in-service lecture followed by one-on-one demonstrations by a radiologist to identify the twinkling artifact before participating in this study.

The urinary tract was evaluated with both gray-scale and color Doppler imaging. The sonographer was blinded to the CT findings and filled out a standardized form indicating the renal size, distance from the skin to the surface of each kidney, body mass index (BMI) of the patient, presence or absence of hydronephrosis, and any areas of high echogenicity with associated shadowing on gray-scale images, the twinkling artifact on Doppler images, or both. Cine clips longer than 2 seconds in duration were also registered for areas of suspected twinkling artifacts to ensure consistency and enable reexamination of any questionable findings.

\section{Comparison of CT and Sonographic Results}

After comparing the two standardized forms (from the independent $\mathrm{CT}$ and independent sonographic evaluations), the radiologist interpreting the CT scan then performed an evaluation of areas in the urinary tract where discordant results were detected between the original CT scan and the blinded sonogram. This evaluation was performed to determine whether a reason for the discrepancy (eg, technical reason on sonography) could be identified. Of note, the radiologist is trained in interpretation of both CT and sonography. The data were analyzed with SPSS version 13 software (SPSS Inc, Chicago, IL) to evaluate the false-positive, true-negative, and false-positive rates of the twinkling artifact as well as the sensitivity and positive predictive value (PPV) of the twinkling artifact. 


\section{Results}

There were 55 patients approached to participate in the study between November 2009 and September 2010; 51 patients enrolled, and 4 declined to participate ( 2 had acute pain, and 2 did not indicate their reasons). There were 17 women and 38 men with average age of 49 years (range, $28-81$ years). The average BMI was $28.4 \mathrm{lb} / \mathrm{in}^{2}$ (range, $16.7-38.3 \mathrm{lb} / \mathrm{in}^{2}$; normal range, $18.5-24.9 \mathrm{lb} / \mathrm{in}^{2}$ ).

The average distances from the skin to the nearest part of the kidney on CT were $5.6 \mathrm{~cm}$ (range, 1.5-10.9 cm) on the right and $5.4 \mathrm{~cm}$ (range, $1.2-11.2 \mathrm{~cm}$ ) on the left. During sonographic evaluation, the sonographer frequently compresses the overlying tissue: the average distances from the skin to the kidney surface were only $4.5 \mathrm{~cm}$ (range, 1.6$6.7 \mathrm{~cm}$ ) on the right and $3.8 \mathrm{~cm}$ (range, $0.9-6.6 \mathrm{~cm}$ ) on the left. The average focal distances used during sonographic evaluation were $7.5 \mathrm{~cm}$ (range, $4.0-11 \mathrm{~cm}$ ) for the right kidney and $7.3 \mathrm{~cm}$ (range, $3.0-12.0 \mathrm{~cm}$ ) for the left kidney.

The average scales (representing the pulse repetition frequency) used to identify the twinkling artifact were 67.7 and $64.1 \mathrm{~cm} / \mathrm{s}$ on the right and left sides, respectively. One hundred fourteen calculi were identified by CT; 13 (23.4\%) patients referred from the ED with flank pain had either other conditions or no clear CT findings to explain the pain. The average size of the calculi as measured on CT was $2.6 \mathrm{~mm}$ (range, $1-9 \mathrm{~mm}$; SD, $1.15 \mathrm{~mm}$ ). Ninety-seven of 114 calculi were less than $5 \mathrm{~mm}$ in diameter on the axial images (85\%). Of the total calculi, there were 35 rightsided and 38 in left-sided renal calculi, 14 right-sided and 21 left-sided ureteric calculi, and 6 bladder calculi. The total numbers of calculi identified in the renal collecting system were 53 on the right and 61 on the left. There were 92 true-positive twinkling artifacts, 6 false-positive artifacts, and 22 false-negative artifacts. In comparison, for gray-scale sonography, there were 74 true-positive findings, 8 falsepositive findings (echogenic structure with shadowing behind it), and 40 false-negative findings for calculi. For gray-scale sonography and the twinkling artifact combined, there were 100 true-positive findings, 4 false-positive findings, and 14 false-negative findings.

The PPV of the twinkling artifact for identifying calculi was $94 \%$ (95\% confidence interval [CI], 87\%-98\%), and the sensitivity was $83 \%$ (95\% CI, $72 \%-87 \%$ ). The PPV of gray-scale sonography for detecting urinary tract calculi was only $64.9 \%$, and the sensitivity was $80.2 \%$. The sensitivity for detecting calculi rose to $88 \%$ when results for both gray-scale sonography (defined as an echogenic structure with shadowing behind it) and the twinkling artifact were combined, and the PPV was $96 \%$.
There was no correlation between the skin-tokidney distance and the ability to identify the twinkling artifact in cases of calculi, which indicates that the twinkling artifact can be identified even in overweight patients $(r=-0.178 ; P=.193)$. Data regarding the BMI were collected, but because fat distribution can be variable, with some patients having more abdominal fat than others, the distance from the skin to the kidney surface was thought to be a more important factor in terms of whether the twinkling artifact could be identified, rather than strictly the BMI.

Of the patients without urinary tract calculi, 2 were found to have renal malignancies as causes of their flank pain; 1 had colitis; 1 had an adnexal mass; and 2 had early diverticulitis. No discrete causes of pain could be identified in the other 7 patients.

\section{Discussion}

Renal colic is a common indication for imaging of patients from the ED. It is estimated that there are more than 1.2 million visits to the ED yearly in the United States with an indication of flank pain, based on national data of $2006 .{ }^{3}$ In addition, during routine abdominal sonography, patients frequently have incidentally discovered echogenic structures in the kidneys, which do not always show shadowing; thus, it can be challenging to definitively say whether these foci represent calculi or not.

Both CT and sonography can be used to identify urinary tract calculi. Computed tomography is generally considered the reference standard, but benefits of sonography include lower cost than $\mathrm{CT}$, the ability to perform the imaging test portably, and no patient exposure to ionizing radiation. One of the major limitations of gray-scale sonography for identifying renal calculi is the presence of small echogenic areas at tissue interfaces, which do not shadow. These interfaces may be false-positively misinterpreted as renal calculi or conversely may obscure visualization of small nearby calculi, leading to false-negative results. Newer-generation ultrasound systems using digital processing technology perceive the twinkling artifact more frequently than older-generation scanners, which used analog technology. ${ }^{4}$ The twinkling artifact is not dependent on the focus, as shown in prior studies. To increase the yield and reproducibility of the twinkling artifact, the examination should be performed with a lowfrequency probe $(2-5 \mathrm{MHz})$ and a high pulse repetition frequency $(>50 \mathrm{kHz}$; Figure 1). In addition, a Doppler tracing showing persistent aliasing should be recorded for longer than 2 seconds (Figure 2). ${ }^{5}$ 
Although unenhanced CT is the reference standard in urolithiasis imaging and has sensitivity of greater than $93 \%$ and specificity of $100 \%$, the slice thickness plays a role in the overall sensitivity. ${ }^{6,7} \mathrm{Jin}$ et $\mathrm{al}^{8}$ showed that the sensitivity of detecting 2- to 4-mm calculi on 5-mm CT slices was only $80 \%$. A retrospective study that assessed the twinkling artifact compared to CT used the 5-mm slice thickness, which carries the inherent possibility of missing smaller calculi ( $85 \%$ of calculi in our cohort were $<5 \mathrm{~mm}$ ). ${ }^{9}$ To overcome this potential limitation, we reviewed the 1.25 -mm raw data in addition to the standard 5-mm reconstructed images. Dillman et $\mathrm{al}^{9}$ retrospectively studied the diagnostic accuracy of the twinkling artifact in assessing nephrolithasis, with an overall PPV of 78\% (95\% CI, 66\%-

\section{A}

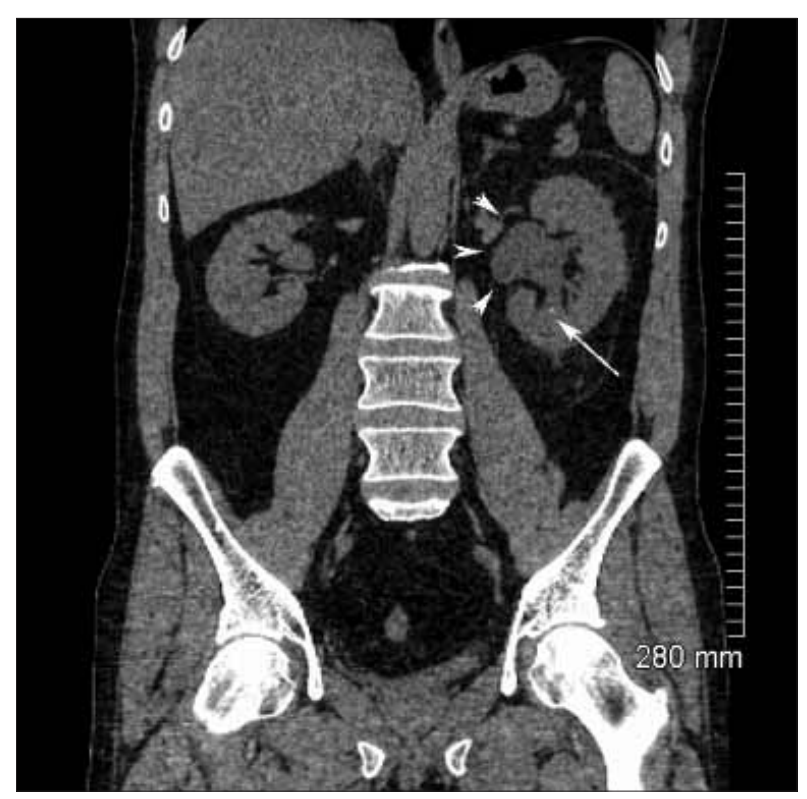

B

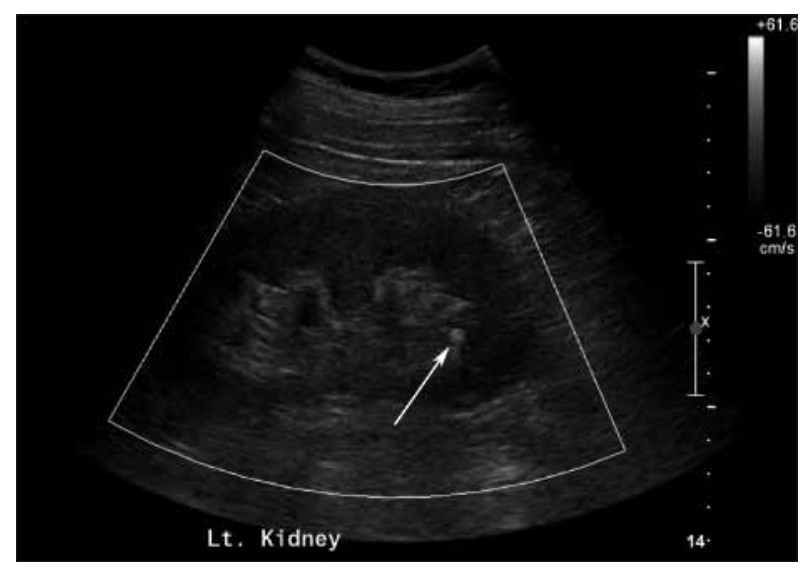

$90 \%)$. The true-positive rate of the twinkling artifact for confirmed calculi on CT was 49\% (73 of 148 twinkling foci), whereas the false-positive rate was 51\% (75 of 148 twinkling foci). Our results indicated a higher PPV for the twinkling artifact, up to $94 \%$ (95\% CI, $0.87 \%-0.98 \%)$ and sensitivity was $83 \%$ (95\% CI, $72 \%-87 \%)$. This difference could be explained by the thinner slice thickness reviewed on CT images, which enabled detection of smaller calculi that could have been claimed as noise and led to incorrect false-positive results on twinkling Doppler images.

Another purported limitation of sonography is that ureteral calculi are not always seen because of overlying bowel gas. ${ }^{10}$ In our study, 5 of 35 ureteral calculi were not detected either by gray-scale sonography or after application of Doppler imaging. This limitation was encountered in calculi located in the mid ureter. Hydronephrosis as a secondary finding of an obstructing ureteric calculus was observed in 2 of the 5 cases, with calculus sizes of 4 and 5 $\mathrm{mm}$, respectively, as seen on CT. In routine clinical settings, this finding would prompt follow-up CT. Further analysis was limited because of the small number of ureteric calculi in the study.

There were 40 false-negative results on gray-scale imaging compared to only 22 false-negative results when the twinkling artifact was assessed. When both gray-scale findings and the twinkling artifact were used together, there were only 14 false-negative results for urinary tract calculi. Grayscale sonography outperformed the twinkling artifact in 5 cases of ureteric calculi at the ureterovesical junction. When

Figure 1. A, Coronal computed tomogram showing hydronephrosis (arrowheads) and a lower calyceal calculus (arrow). B, Corresponding gray-scale sonogram showing an echogenic structure corresponding to a calculus seen on CT. C. Color Doppler sonogram of the left kidney, with a high pulse repetition frequency $(61.6 \mathrm{~cm} / \mathrm{s})$, showing the area of the twinkling artifact.

C

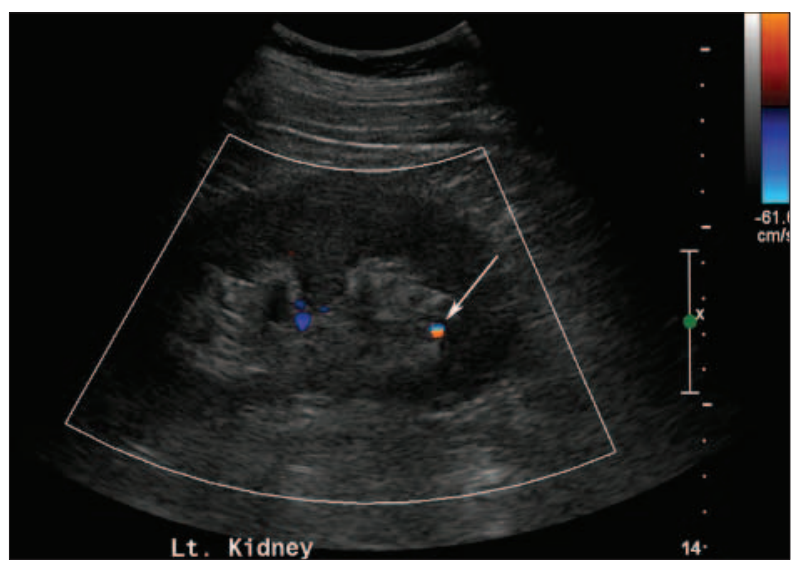


we reviewed the sonographic parameters, we observed a low pulse repetition frequency $(38 \mathrm{~cm} / \mathrm{s})$ for 2 cases, which may have accounted for these false-negative twinkling results. The remaining 3 cases had optimized settings for the pulse repetition frequency and focal spot. The reason for this observation is not clear and could be attributed to the calculus composition or smoothness of the surface (Figure 3). We were not able to analyze the calculi subsequently.

There were 21 cases of urinary tract calculi in which the twinkling artifact was present and corresponded to a calculus on CT, but gray-scale sonography could not identify the calculi well. In some cases, during the subsequent unblinded review by the radiologist who interpreted the original CT scans, the calculi could be subtly identified in retrospect after the color Doppler mode was turned off.

There were 6 false-positive findings of the twinkling artifact. In 1 case, there were 2 pelvic phleboliths that showed the twinkling artifact, but it was clear from the sonograms that these were not near the ureter; thus, in a clinical situation, this appearance would not be misinterpreted (Figure 4). Therefore, the combination of the twinkling artifact and gray-scale images had only 4 false-positive findings. Another case of a false-positive twinkling artifact in the kidney was thought to represent some very early changes of calculus formation based on a retrospective review of the $\mathrm{CT} \operatorname{scan}(1.25$-mm cuts). This case may have represented a false-negative CT result. Another false-positive twinkling artifact occurred in the same patient; it occurred next to a small angiomyolipoma seen on CT (Figure 5). It was still classified as a false-positive finding. There was 1 false-positive vascular calcification in a kidney that showed the twinkling artifact in a 48-year-old woman with diffuse atherosclerosis. Other hypothesized potential

Figure 2. Doppler tracing of longer than 2 seconds (bottom) required to confirm a true area with a twinkling artifact in the lower pole of the kidney (arrow). This tracing confirms that the twinkling artifact is not simply due to motion of the probe over an area of heterogeneous tissue.



causes of false-positive twinkling artifact results could include milk of calcium in a cyst, although there were no such cases in our cohort. It is possible that some of the twinkling artifact findings on sonography were actually correct, but the calculi were either minuscule or of variable composition (low concentration of calcium) and thus were not identified on CT, even in retrospect. Using low-dose renal colic $\mathrm{CT}$ scan protocols can lead to noisy images, particularly in larger patients. ${ }^{11}$

We calculated the BMI for patients based on their height and weight but also measured the distance from the skin to the kidney on $\mathrm{CT}$ and sonography as predictive factors for detecting the twinkling artifact. Because patients have different distributions of fat, we thought that the BMI was not a sufficient measure for determining whether it

Figure 3. False-negative twinkling artifact in a 56-year-old man with right-sided pain. A, Gray-scale sonogram showing an echogenic obstructing calculus (arrow) in the distal right ureter. B, Despite optimization of parameters (high pulse repetition frequency), there is no corresponding twinkling artifact on the color Doppler sonogram (arrow).

A

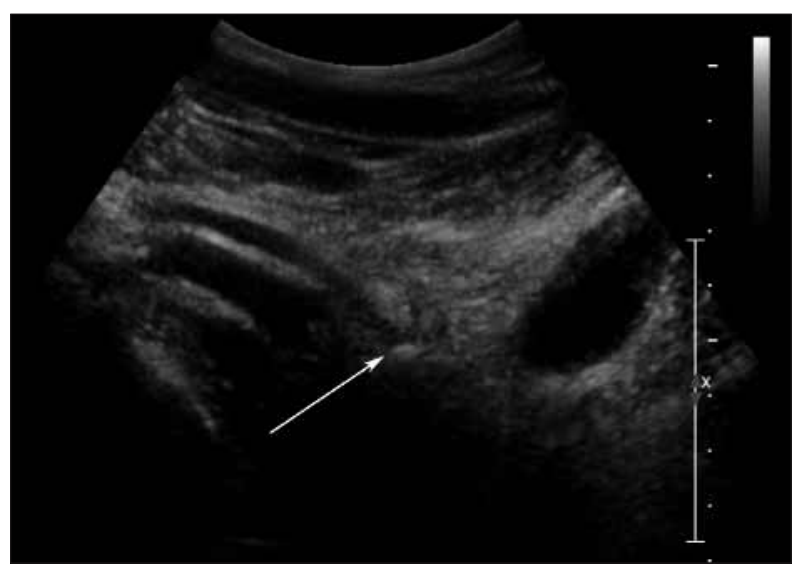

B

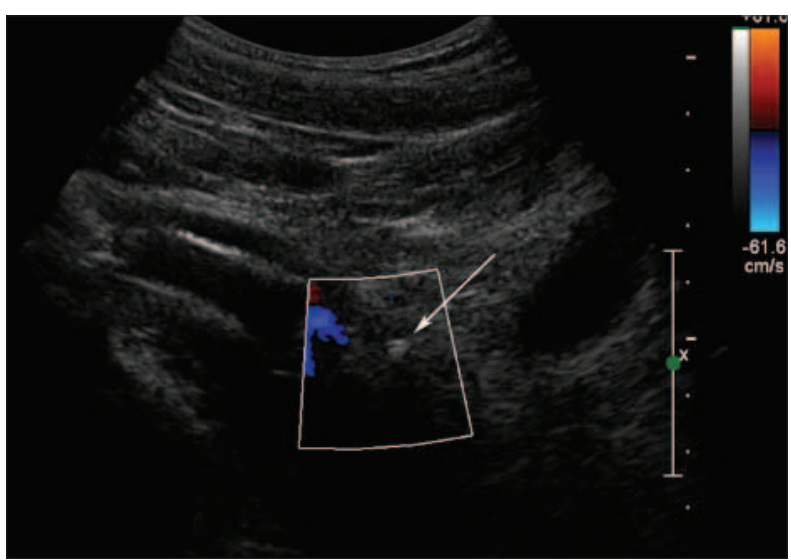


affected visualization of the twinkling artifact. As determined by the Spearman $\rho$ nonparametric test, there was no correlation between the BMI or the distance from the skin to the kidney on sonography with respect to detecting the twinkling artifact.

As part of this study design, the ED physicians were not involved to reduce referral bias. Thus, if a sonographic examination was requested from the $\mathrm{ED}$, we only performed the examination and did not enroll the patient in our study. This process would explain the fairly high average age of the patients in this study. Although a few younger patients were included in our study cohort (mostly with higher BMIs), in our institution, most young patients are typically evaluated first with sonography.

Figure 4. False-positive twinkling artifact in a patient with pelvic phleboliths. A, There is a focus of a twinkling artifact shown in the left lower quadrant (arrow). B. On the subsequent image, however, there is a normal ureteric jet (arrow) in a separate location from the area of the twinkling artifact, indicating that these are pelvic pheloboliths rather than renal calculi.

A

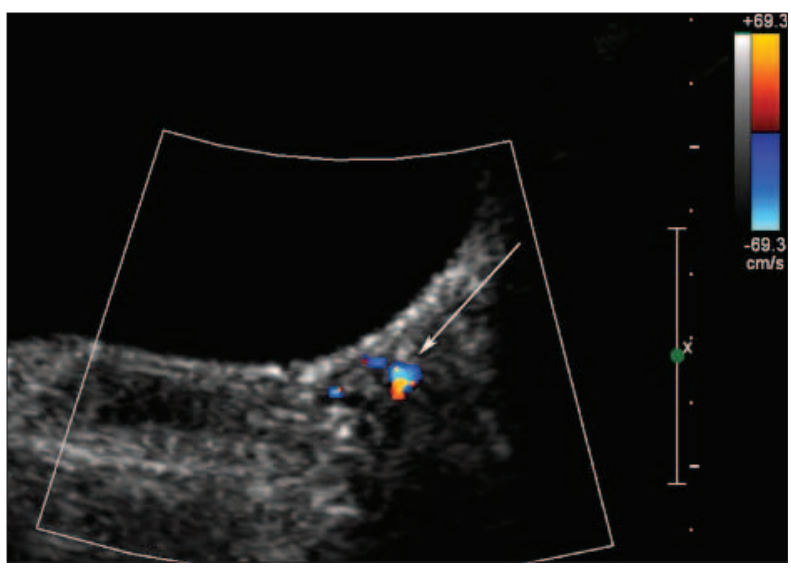

B

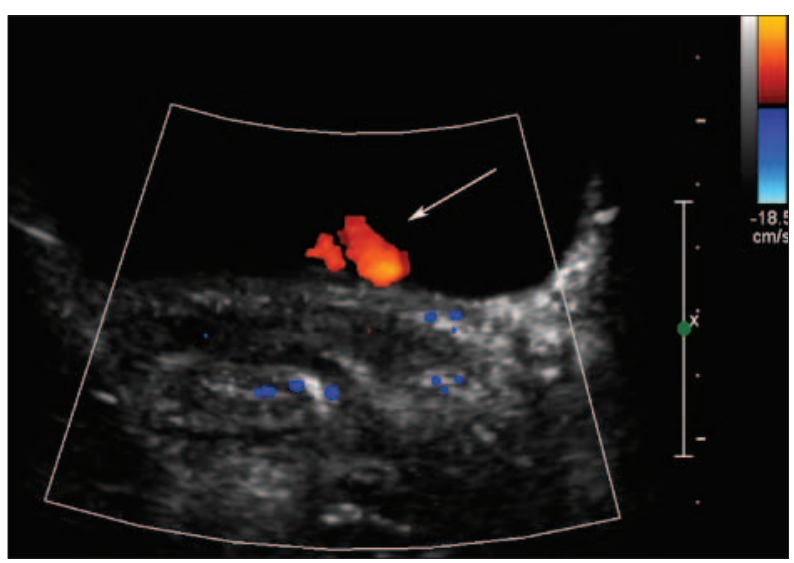

One of the limitations of this study was that the patient population was not sequential. We perform CT scans in the ED on a 24-hour basis, but the radiologists involved in the study were not available at all hours. Nevertheless, the radiologists did not see or interact with the patients before the decision to enroll them in the study; thus, patient selection bias should have been low.

Another weakness of the study was that the calculi were not collected from the patients after they passed; thus, we could not determine whether the cause of a falsenegative twinkling artifact was due to the shape (ie, rough or irregular surface) or due to the composition of the calculi. However, most urinary tract calculi ( $80 \%)$ contain calcium and are therefore readily visible on CT and would be

Figure 5. A, Computed tomogram from a 50-year-old woman showing a small angiomyolipoma in the lower right kidney (arrow). B, Corresponding Doppler sonogram showing an area with a twinkling artifact (arrow) next to the angiomyolipoma (arrow). The echogenic angiomyolipoma is not shown on the sonogram.

\section{A}

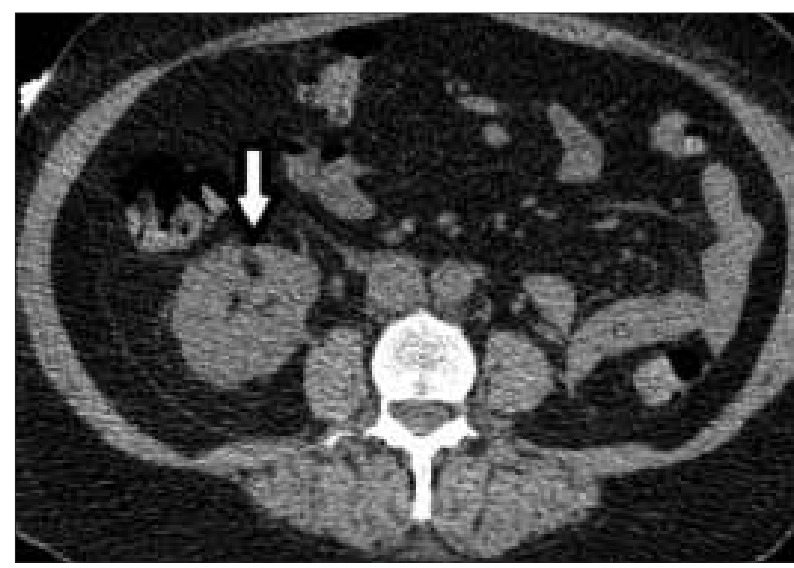

B

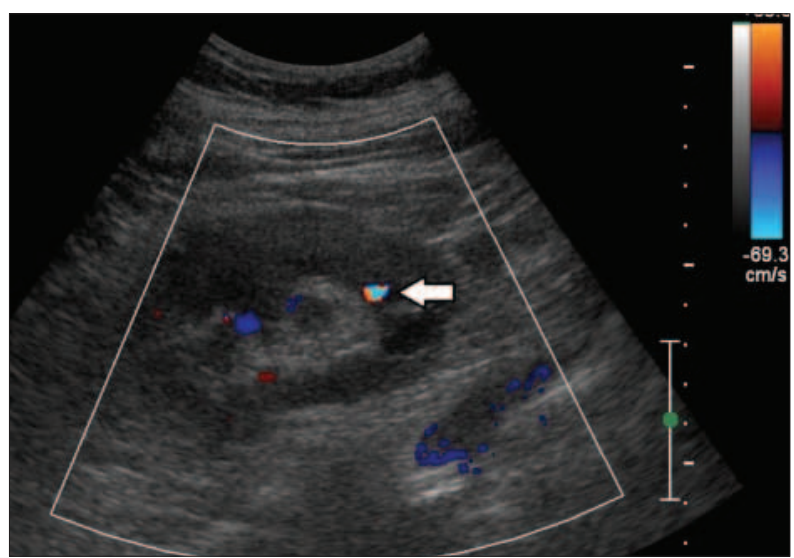


expected to be reflective on sonography. ${ }^{12}$ Other more rare calculi include struvite calculi, zidovudine calculi, and cysteine calculi, which may not be as reproducibly shown on sonography, although this issue has not been extensively studied. ${ }^{12}$

In conclusion, the twinkling artifact, when imaged with a high pulse repetition frequency, can show nonobstructing calculi as well as many obstructive calculi. Evaluation for the twinkling artifact is a complementary technique to standard gray-scale shadowing of calculi and improves detection of urolithiasis on sonography. However, if a calculus is not definitely seen in an acute setting of flank pain, the presence of hydronephrosis on the side of the pain should prompt a follow-up CT scan if clinically indicated.

\section{References}

1 Rahmouni A. Bargoin R, Herment A, Bargoin N, Vasile N. Color Doppler twinkling artifact in hyperechoic regions. Radiology 1996; 199:269-271.

2. Trillaud H, Pariente JL, Rabie A. Grenier N, Detection of encrusted indwelling ureteral stents using a twinkling artifact revealed on color Doppler sonography.AJR Am J Roentgenol 2001; 176:1446-1448.

3. Pitts SR, Niska RW, Xu J, Burt CW. National Hospital Ambulatory Medical Care Survey: 2006 emergency department summary. Natl Health Stat Rep 2008; 7:1-38.

4. AytaçSK, Özcan H. Effect of color Doppler system on twinkling sign associated with urinary tract calculi.J Clin Ultrasound 1999; 27:433-439.

5. Kamaya A, Tuthill T, Rubin JM. Twinkling artifact on color Doppler sonography: dependence on machine parameters and underlying cause. AJR Am J Roentgenol 2003; 180:215-222

6. Niemann T, Kollmann T, Bongartz G. Diagnostic performance of lowdose CT for the detection of urolithiasis: a meta-analysis. AJR Am J Roentgenol 2008; 191:396-401.

7. Ripollés T, Agramunt M, Errando J, Martinez MJ, Coronel B, Morales M. Suspected ureteral colic: plain film and sonography vs unenhanced helical CT — a prospective study in 66 patients. Eur Radiol 2004; 14:129_ 136.

8. Jin DH, Lamberton GR, Broome DR, et al. Renal stone detection using unenhanced multidetector row computerized tomography: does section width matter? J Urol 2009; 181:2767-2773.

9. Dillman JR, Kappil M, Weadock WJ, et al. Sonographic twinkling artifact for renal calculus detection: correlation with CT. Radiology 2011; 259:911-916.

10. Gaspari RJ, Horst K. Emergency ultrasound and urinalysis in the evaluation of flank pain. Acad Emerg Med 2005; 12:1180-1184.

11. Kim BS, Hwang IK, Choi YW, et al. Low-dose and standard-dose unenhanced helical computed tomography for the assessment of acute renal colic: prospective comparative study. Acta Radiol 2005; 46:756-763.

12. Moe OW. Kidney stones: pathophysiology and medical management. Lancet 2006; 367:333-344. 